\title{
Physicochemical Study of Viral Nanoparticles at the Air/Water Interface
}

Jose F. Torres-Salgado, ${ }^{l}$ Mauricio Comas-Garcia, ${ }^{2}$ Maria V. Villagrana-Escareño, ${ }^{l}$ Ana L. Durán-Meza, ${ }^{1}$ Jaime Ruiz-García ${ }^{1}$ * and Ruben D. Cadena-Nava., *

${ }^{1}$ Instituto de Física, Universidad Autónoma de San Luis Potosí, Álvaro Obregón 64, 78000 San Luis Potosí, SLP, México.

2 HIV Dynamics and Replication Program, National Cancer Institute, Frederick, Maryland 21702, United States

3 Universidad Nacional Autónoma de México, Centro de Nanociencias y Nanotecnología. Carretera Tijuana-Ensenada Km. 107, 22860 Ensenada, B.C. México.

*Authors to whom correspondence should be addressed. E-mail: rcadena74@gmail.com (R.D.C.-N.), jaime@dec1.ifisica.uaslp.mx (J.R.-G.).

\section{SUPPLEMENTARY MATERIAL}

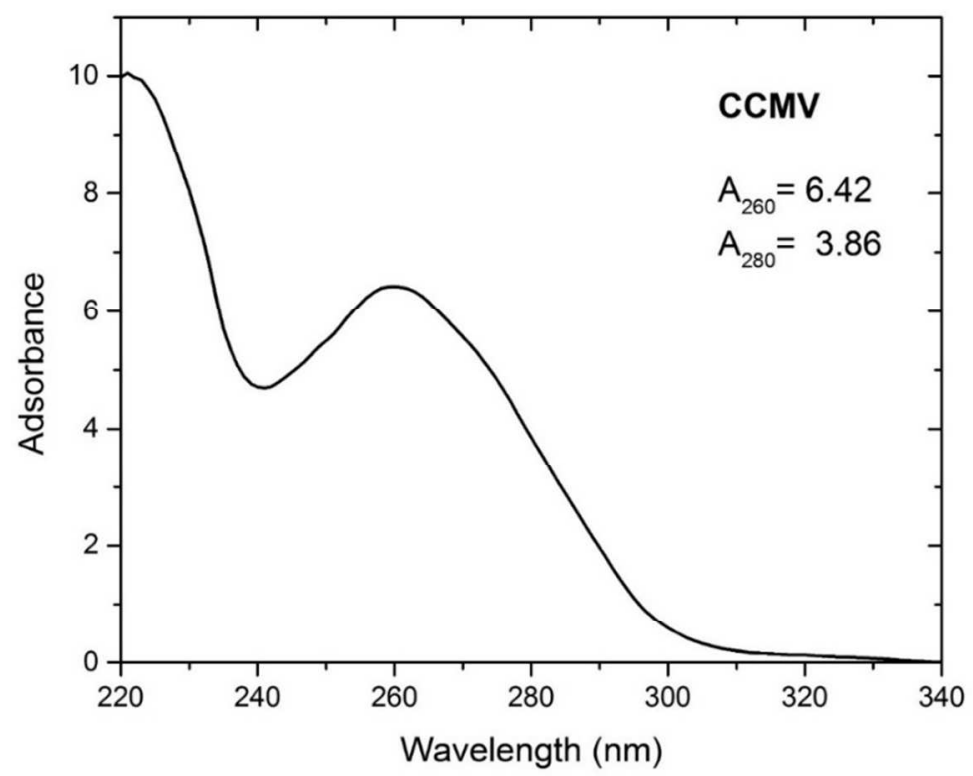

Figure S1. Typical UV absorption spectrum of wild type CCMV in buffer B obtained by Nanodrop UV-vis spectrophotometer. The peak at $260 \mathrm{~nm}$ corresponds to the maximum absorbance of CCMV ssRNA and the capsid proteins have a contribution at $280 \mathrm{~nm}$. A high purity of CCMV is considered for the absorbance ratio $A_{260} / A_{280} \geq 1.5$. For this UV spectrum the purity of CCMV is 1.66 . 
Table S1: The purity of CCMV capsid protein is determined according to the percentage of nucleic acid contamination in the solution by the absorbance ratio $\mathrm{A}_{280} / \mathrm{A}_{260}{ }^{45} \mathrm{~A}$ good quality of CCMV CP is considered for value ratios $\geq 1.5$, which correspond to values $<0.5 \%$ of nucleic acids as contaminants.

\begin{tabular}{|c|c|c|}
\hline $\mathrm{A}_{280} / \mathrm{A}_{260}$ & \% nucleic acids & $\begin{array}{c}\text { Cell factor for } \\
\text { cm path length }\end{array}$ \\
\hline 1.75 & 0.00 & 1.116 \\
\hline 1.63 & 0.25 & 1.081 \\
\hline 1.52 & 0.50 & 1.054 \\
\hline 1.40 & 0.75 & 1.023 \\
\hline 1.36 & 1.00 & 0.994 \\
\hline 1.30 & 1.25 & 0.970 \\
\hline 1.25 & 1,50 & 0.944 \\
\hline 1.16 & 200 & 0.899 \\
\hline 1.09 & 2.50 & 0.852 \\
\hline 1.03 & 3.00 & 0.814 \\
\hline 0.979 & 3.50 & 0.776 \\
\hline 0.939 & 4.00 & 0.743 \\
\hline 0.874 & 5.00 & 0.682 \\
\hline 0.846 & 5.50 & 0.658 \\
\hline 0.822 & 6.00 & 0.632 \\
\hline 0.804 & 6.50 & 0.607 \\
\hline 0.784 & 7.00 & 0.585 \\
\hline 0.767 & 7,50 & 0.565 \\
\hline 0.753 & 8.00 & 0.545 \\
\hline 0.730 & 9.00 & 0.508 \\
\hline 0.705 & 1.00 & 0.478 \\
\hline 0.671 & 12.00 & 0.422 \\
\hline 0.644 & 14.00 & 0.377 \\
\hline 0.615 & 17.00 & 0.322 \\
\hline 0.595 & 20.00 & 0.278 \\
\hline & & \\
\hline
\end{tabular}

